Revue scientifique francophone en Communication

organisationnelle

$18 \mid 2000$

Non-verbal, communication, organisation

\title{
La communication inter personnelle intéractive et l'expression du corps
}

\section{Michèle Gabay}

\section{OpenEdition}

\section{Journals}

Édition électronique

URL : https://journals.openedition.org/communicationorganisation/2408

DOI : 10.4000/communicationorganisation.2408

ISSN : 1775-3546

\section{Éditeur}

Presses universitaires de Bordeaux

\section{Édition imprimée}

Date de publication : 1 novembre 2000

ISSN : 1168-5549

Référence électronique

Michèle Gabay, "La communication inter personnelle intéractive et l'expression du corps »,

Communication et organisation [En ligne], 18|2000, mis en ligne le 27 mars 2012, consulté le 05 août

2021. URL : http://journals.openedition.org/communicationorganisation/2408 ; DOI : https://doi.org/

10.4000/communicationorganisation.2408

Ce document a été généré automatiquement le 5 août 2021 .

(C) Presses universitaires de Bordeaux 


\title{
La communication inter personnelle intéractive et l'expression du corps
}

\author{
Michèle Gabay
}

1 Le corps par nos attitudes, nos gestes détachés ou non détachés du discours nous renseigne-t-il sur l'interaction dans un processus de communication? Cette recherche couvre une partie de la communication interpersonnelle, j'entendrai par interaction: l'articulation d'un échange qui met deux individus en situation physique de face à face. Cette articulation témoigne d'une interrelation qui passe par des signes multiviatiques, que les protagonistes sont capables alternativement d'émettre et de percevoir de façon consciente ou inconsciente. L'interaction provoque, chez les partenaires, des changements mutuels dans leur comportement, voire dans leurs attitudes.

2 Quels sont les processus mis en jeu? Quelles fonctions remplit la communication interactive et interpersonnelle CII? Quels types de communication? Comment le message est-il communiqué et interprété ? La recherche des indicateurs de l'interaction dans ce processus de communication m'a conduite à m'interroger sur leur observation et leur consignation.

3 La méthodologie utilisée est «l'observation croisée». Plusieurs situations ont été observées par la médiation d'un caméscope. Il s'agit de situations de simulations d'études de cas avec résolution de problème ou de situations de travail réelles filmées comme par exemple des réunions.

\section{La communication interactive interpersonnelle}

\section{Le concept}

4 Dans la communication interactive (pas au sens donné dans la communication médiatique informatique, définie par Lippman (1989) et interpersonnelle, il y a échange de messages linguistiques et non linguistiques (images, gestes, sons), entre un individu émetteur $\mathrm{E}$ et un ou plusieurs autres individus récepteurs $\mathrm{R}$ (nous gardons cette terminologie traditionnelle pour plus de commodité). Cet échange transmet des 
informations et/ou provoque un changement. Le changement se traduira par un comportement tel que l'E, à son tour touché par cette manifestation, modifiera son comportement, voire son attitude. Cette influence reste une boucle permanente dans une interrelation entre l'E et le R. Dans le contexte de CII, tout comportement est, à la fois, réponse et stimulus; ils dépendent de l'état de l'E ou/et du R, et de la situation de mise en communication.

5 Le changement devra être étudié en fonction de toutes les variables et de tous les acteurs. Cependant, il est difficile dans la CII, voire impossible, de neutraliser l'effet d'un facteur pour en mesurer un autre. Deux autres réserves: la résistance au changement, et le leurre de l'apparence d'un changement ; un individu, menacé dans les structures profondes de sa personnalité, oppose des barrières de protection inconscientes qui l'empêchent de changer. Enfin l'expression orale d'un changement, ne signifie pas forcément un changement d'opinion profond suivi d'un changement d'action (Gabay. 1978).

La communication n'est pas interactive, lorsqu'il n'y a pas d'interrelation entre l'E, et le ou les R, lorsqu'il manque un facteur, le retour (ou feed-back), qui donne la possibilité de réajustement tant à l'E qu'au $\mathrm{R}$, par exemple si les partenaires ne sont pas en présence l'un de l'autre.

\section{Processus Je la communication interactive interpersonnelle}

Aujourd'hui, la position la plus fréquemment admise en ce qui concerne le processus de la CII, s'articule autour d'un système où les éléments sont à la fois dépendants et complémentaires.

8 La CII est un système social commun qui met en scène une ou plusieurs personnes, dont chacune élabore par rapport à l'autre un projet d'échange qui comprend des attentes, des enjeux et des intentions. Le système est sous-tendu par des interrelations sociales d'ordre affectif, de pouvoir symétrique, dissymétrique, d'intimité, de connivence etc.

Ce système s'élabore dans un processus dynamique bouclé où, au cours de la séquence, chaque intervention, tant de l'E que du R, s'influence l'une et/ou l'autre. Même sans influence souhaitée et quelle que soit l'interprétation des partenaires, il se produira un changement. La CII exerce un pouvoir de changement dans le comportement immédiat, mais parfois plus profond dans les attitudes. Les interventions sont interdépendantes dans le temps.

10 La CII est multiviatique ou multicanaux (Gabay, 1990), chaque individu émet ou reçoit un message total qui résulte de la combinaison synergique d'éléments vocoacoustiques, visuels, olfactifs et tactiles. Les différents sens qui servent à la communication des hommes empruntent différents canaux. Le canal auditif véhicule le langage verbal qui utilise un code, la parole. Le canal visuel permet d'appréhender le langage du corps en fonction des postures ou des attitudes, indicateurs statiques, ou bien par la cinétique, les mouvements, la mimogestualité et par la proxémique, l'évaluation visuelle des espaces, des distances. Hubert Montagner (1978) a constaté une identification par l'odeur. Enfin le canal tactile et thermique met en évidence la communication par le contact de la peau, de la surface du corps celui-ci recouvre toute une dimension de la communication: depuis les liens sociaux, exemple, poignée de main, jusqu'aux gestes de séduction et d'amour. Je déplore le fait de privilégier ou de « gommer » un des canaux, chacun pouvant neutraliser, annuler, modifier, minimiser 
ou maximiser les effets de l'autre, pour moi la communication est multiviatique. Nos cinq sens s'unissent, s'enchevêtrent et créent une relation entre la sensorimotricité et la parole. "Chaque sensation sous-tend une activité motrice réelle ou virtuelle» (Boulanger, Balleyguier, 1967)

11 Ce système est non autonome, il existe à un moment donné, dans un environnement particulier, tant physique que social.

\section{Les différents types de Communication Interactive Interpersonnelle}

12 La communication consommatoire selon Festinger (1950) se traduit chez un individu, par l'extériorisation corporelle d'une émotion, d'une motivation ou d'une activité cognitive qui ne serait pas destinée à un R. Un individu, sans en avoir l'intention et souvent même à son insu, renseigne sur ses besoins, ses envies, ses ressentiments, ses intérêts etc.. (Zajonc, 1972) Ces communications sont dites incidentes.

La communication instrumentale ne prend consistance qu'en présence d'un tiers à qui elle est destinée. Elle est anticipée et contrôlée en fonction du

$\mathrm{R}$, et suivant la réponse que l'E attend de lui. Les individus communiquent et souhaitent communiquer.

Quelle que soit la communication, cela n'implique pas que la production d'expressions soit une mise en relation claire et mono sémique entre des individus qui communiquent. Certes, il existe des règles de productions langagières partagées par un groupe social, mais il n'est à aucun moment possible de maîtriser des interprétations.

\section{Les fonctions de la CII}

Elles s'analysent dans l'interrelation. La CII peut avoir pour but de modifier l'état du destinataire ; de sa connaissance du sujet en l'informant. L'E peut faire changer d'avis le $\mathrm{R}$ par la persuasion. Il peut aussi améliorer son état affectif.

La CII peut viser à sauvegarder ou à changer l'état des relations interpersonnelles afin de les réguler ou de les rétablir.

Elle peut avoir pour but de faire agir le destinataire, en déclenchant chez lui une action en réponse à un ordre, une demande, une prière...

19 Elle peut servir à l'affirmation de soi, à assurer son pouvoir par exemple Berkowitz (1975) ; dans le but de rehausser l'estime de soi auprès des autres.

20 Particulièrement dans la CII plusieurs fonctions peuvent se superposer la voix par le ton, le rythme, le débit et toutes les attitudes corporelles la complexifient. aussi convient-t-il d'analyser à chaque fois, tous les codes du langage de l'interaction.

\section{Observation des attitudes corporelles.}

\section{Le corps médiateur de la communication}

21 Ma recherche porte sur le canal auditif et le canal visuel. Les éléments verbaux et non verbaux coexistent avec des redondances, des suppléances et des dissociations. 


\section{l'observation de la communication qui revêt une fonction expressive, tant du point de} vue de l'E que de celui du R, elle est impressive, communicative et heuristique.

\section{Observation des interactions}

La méthode d'observation utilisée pour cette recherche est «l'observation croisée » avec utilisation d'un caméscope. Dans toutes les situations de CII enregistrées au caméscope, puis visionnées ensuite chaque sujet observé fait l'objet d'une triple observation, la sienne par l'autoscopie, l'individu s'observe lui-même à partir de l'enregistrement au caméscope d'une séquence qu'il visionne, il est $\mathrm{E}$ et $\mathrm{R}$ à la fois, celle d'observateurs internes ou externes à la situation de CII et celle du formateur (non étudiée dans cet article).

Les observations ont porté sur plusieurs centaines d'enregistrements. Les attitudes corporelles traduisant les émotions, l'attention, la compréhension ont été observées à travers le regard, les gestes, détachés ou accompagnant le discours, les mimiques, les silences, les apartés. Enfin, le changement a été évalué par l'observation de l'action ou la simulation d'action au cours d'exercices. Les observateurs consignent leurs observations sur des grilles élaborées en fonction des objectifs, soit par le groupe de formés, soit par moi.

visionnement au caméscope permet de dissocier les étapes de l'analyse d'une situation de communication. Par «l'observation croisée » les observateurs et l'observé peuvent établir une liaison entre le signifiant et le signifié, et vérifier comment s'est effectué le feed-back. J'ai pu noter que l'observation des gestes dans un premier temps est parcellisée et quantitative, puis de descriptive elle devient ensuite qualitative et interprétative. Les «observations qualité » ou «observations interprétation " font découvrir un code, mais aussi une polysémie des gestes et des attitudes. De plus les observateurs découvrent des significations trahissant les structures plus profondes de la personnalité.

Lorsque l'observateur est extérieur à l'interaction, il observe et comprend le processus de l'interaction. Il va voir que les acteurs suivent une règle de l'alternance, dans leurs interventions, il essaie d'identifier un modèle de redondance. Il interprète le sens. Son explication part d'hypothèses et aboutit à des significations profondes, réelles ou fantasmatiques.

27 L'observateur qui s'observe lui-même essaie d'analyser le modèle d'interaction qu'il poursuit. Il peut être conscient des règles de comportement qu'il adopte, ne pas en être conscient, mais être capable de les identifier lorsqu'un tiers les lui signale. Enfin, il peut se montrer incapable de voir, d'admettre, ses comportements, même si on les lui signale, tellement ils sont éloignés de sa conscience.

28 Le phénomène de projection intervient dans la communication et dans l'observation, c'est un phénomène de résonance inconscient lié à l'affectivité du sujet et à l'expérience cognitive de vie de l'individu. Tant l'E que le R, projette son vécu dans la perception et dans l'appréhension d'un message. Le phénomène de projection induit des attitudes qui perturbent et nuisent à la CIL (Gabay, 1990) Une observation avec distanciation affective temporelle permet la prise de conscience de ce phénomène, l'observation avec utilisation du caméscope établit cette distanciation. Le sujet s'observe lui-même en autoscopie, il est sujet et objet de l'observation. L'individu est

Communication et organisation, 18 | 2000 
capable de se projeter dans l'espace virtuel : c'est l'objectivation. L'évolution de la prise de conscience de soi se définit selon les processus de distanciation, puis d'intégration par rapport au vécu initial. Pour que l'individu se comprenne comme sujet, il doit se voir comme objet, l'autoscopie permettant de se voir comme objet l'autorise à se comprendre comme sujet. Le phénomène d'objectivation se trouve renforcé par le travail «d'observation croisée ». La distanciation sera facilitée, d'une part, grâce aux performances techniques du caméscope qui assurent de pouvoir filmer de l'instantané puis de le visionner et d'autre part, grâce à l'introduction d'une personne extérieure à l'événement qui en quelque sorte objective l'observation (Gabay, 1978)

La distanciation sensibilise aux phénomènes individuels, et aux phénomènes d'interaction dans les groupes.

Par ailleurs, l'enregistrement d'une séquence de travail au caméscope, introduit une distanciation temporelle : l'observateur possède un témoignage visuel et auditif, qu'il peut voir et revoir avec des entrées de lectures diverses ce qui permet de sélectionner le flot d'informations visuelles et auditives et de les interpréter. L'observation croisée sensibilisera à l'évaluation d'un système de relation ou de non-relation, existant entre la codification du signifiant et du signifié.

31 Cependant le cameraman, parce qu'il filtre déjà l'information qu'il filme introduit un biais.

\section{Les indicateurs}

Pour nourrir cette analyse je me suis basée, sur les signaux para linguistiques liés à l'émission sonore, à la voix, l'intensité, la hauteur de ton, les pauses, la pose de la voix, l'accent, le rythme et des vocalisations comme les rires, les cris, les grognements, onomatopées et sur les signaux non linguistiques comme les gestes, les expressions faciales, les mimiques, les regards, l'occupation de l'espace. Ces signaux prennent la forme de comportements non verbaux, ils constituent des indicateurs à partir desquels les participants à la CII font des inférences et s'attribuent l'un à l'autre des intentions, des dispositions définitives ou temporaires qui soutiennent la CII. Les indicateurs transitent, en quelque sorte, par un code qui est une combinaison de signes. Dans une société culturelle donnée, beaucoup de signes ont une signification partagée. Cependant, le répertoire de signes n'est pas mono sémique. Les signes du code sont arbitraires même s'ils renvoient à des réalités conceptuelles, vécues ou imaginaire. De plus, même un code commun, n'induit pas systématiquement une authentique CII.

Les gestes dénotatifs correspondent à la gestualité symbolique dans le sens des gestes ritualisés. La connotation des gestes est l'expression des affects, des émotions elle intègre la dimension cognitive. Pour Fraisse (1974), Ekman et.Friesen (1981) les émotions se manifestent d'une façon privilégiée sur le visage, véritable plaque sensible, et les mains.

Les apports paléontologique et ethnologique, Leroi-Gourhan (1975) démontrent que l'emploi simultané de la main et de la face se trouve dans le comportement d'un nombre important d'espèces depuis les origines. La main, le visage et la posture de préhension sont directement liés à la construction corporelle de l'homme. Il se singularise des autres espèces car il n'utilise que les membres antérieurs pour l'acte de préhension qui ne lui servent pas à se déplacer. Les gestes des mains et les expressions de la face accompagnent la communication verbale dans l'exercice du langage. 11 faut 
constater une coordination des gestes et de la parole; ainsi, les gestes de la main accompagnent le discours comme un commentaire de la parole. La fonction de symbolisation se trouve, tant dans l'expression verbale, que dans l'expression écrite assurée par l'intermédiaire de la main. (Barrier, 1998). anthropoïdes et l'homme, sont en corrélation avec la libération des mains et de la bouche, qui ne servent plus à la survie. Chez l'enfant, l'évolution des gestes obéit à un schéma programmé. Jusqu'à un an, l'enfant imite les gestes des personnes; ensuite, il mime les objets, puis la maturation opère une individualisation des gestes en fonction de son tempérament, de ses aspirations. Le nourrisson structure sa personnalité et développe sa capacité à communiquer, à travers le vécu de ses interactions nonverbales avec sa mère, il s'agirait même d'un héritage phylogénétique. nourrisson vit une interrelation avec sa mère, il sourit, gazouille en réponse à ses expressions. Les expressions s'enrichissent ensuite du fait des besoins de la 
communication avec les autres, et certaines mimiques n'existent que si nous communiquons avec d'autres personnes. Selon Erving

Goffman (1973) les comportements des individus dans un groupe pris au sens large comptent des relations anonymes et des relations ancrées.

Certains gestes apparaissent suivant un programme, mais les exigences sociales peuvent les régler. L'individu apprend à réprimer, libérer ou même simuler des comportements selon les besoins de la situation sociale. Lorsque cette situation se prolonge ou se reproduit fréquemment, cela devient un mode de fonctionnement expressif naturel pour l'individu.

La part de socialisation des gestes est considérable M. Mauss (1936) a établi des déterminants culturels dans les façons dont les hommes savent se servir de leur corps. Il y a une sorte de transmission traditionnelle, dans une société, des façons de remplir les fonctions les plus fondamentales. Certains gestes sont conditionnés par l'apprentissage familial ou scolaire ou sont guidés par l'imitation spontanée d'êtres aimés ou de personnes admirées.

Le groupe social dans lequel vivent des individus, modèle certains gestes et comportements qui peuvent à première vue apparaître comme naturels, innés et caractériser à tort une race Efron (1972). Chaque groupe, depuis la petite cellule familiale jusqu'à la nation, possède ses règles, ses habitudes, ses conventions gestuelles qui accompagnent, précèdent ou remplacent le langage verbal. Ces gestes sont parfois ritualisés, Goffman (1988), ils correspondent à une répétition automatique d'un modèle antérieur socialement déterminé. Il en résulte qu'au sein d'un même groupe beaucoup de gestes peuvent être interprétés par rapport à un code social. Toutes les personnes émettent des messages non verbaux par leurs gestes, leurs attitudes, leurs postures, leurs façons de se maquiller, de s'habiller qui renseignent les autres sur l'âge, le statut et le rôle.

6 La psychanalyse ne néglige pas les comportements corporels. Tous les événements de notre vie affectivo-caractérielle, de notre propre vécu instinctuel, émotionnel et relationnel, laissent une trace dans l'état permanent et le mode de fonctionnement de notre tonicité Le Camus (1975). Freud avait mis en relief la signification psychanalytique de certains actes manqués ou de ratés du geste. Pour Lebovici (1952), les tics sont l'expression d'un conflit. Beaucoup de thérapeutes se servent des gestes ou attitudes corporelles comme diagnostic ou comme thérapie. Nous retiendrons que. pour les psychanalystes, le corps s'envisage comme un fantasme produit par l'imaginaire, et signifié par un langage, et certains gestes pourront avoir une résonance inconsciente.

47 L'approche psychologique s'attache aux gestes dans l'évolution de la personnalité et dans les aspects dynamiques des manifestations psychiques.

Quelques traits de la personnalité apparaissent dans des attitudes de la personne suivant l'espace qu'elle occupe, l'extraverti, occupe tout son espace intime, avec des gestes larges. L'introverti, a des attitudes plus raides, plus bloquées, des mouvements plus courts, il tente de ne pas se faire remarquer, il essaie d'occuper le moins d'espace possible. Nos gestes trahiraient notre personnalité psychique qui serait liée, dans ses apparences, et dans ses profondeurs, à notre personnalité physique. Sheldon affirme que notre personnalité physique trouve ses soubassements dans notre morphologie qui déterminerait notre tempérament, ce tempérament s'exprimant par des attitudes, des 
gestes. Il convient d'être critique dans ces interprétations, il y a certainement une relation entre les stéréotypes, préjugés de l'imagination populaire et «le tempérament ». Cependant, la constitution physiologique imposera dans certains cas des attitudes gestuelles.

Certains états psychiques déclenchent des réactions corporelles. L'émotion provoque la mise en tension du corps. En effet l'organisme se prépare à l'action, en anticipant sur les modèles antérieurs de réaction construits par l'expérience ou l'imitation d'autrui. Les gestes de réaction sont, à la fois, imprimés en nous par un code social, et tempérés par notre personnalité qui censure ou laisse apparaître nos sentiments.

En conclusion nos gestes disent, en partie, ce que nous sommes, à travers la situation que nous vivons, et la façon que nous avons de la vivre à un moment donné. Tous les gestes, dans la mesure où ils expriment quelque chose sont des éléments de communication, même s'ils ne sont porteurs que d'une information. Les gestes apparaissent comme un système de signes socialisé propre à l'individu comme sujet avec sa construction corporelle unique accompagnant les autres langages dans la communication. Chaque individu compte des patterns mimogestuels spécifiques. Enfin quelles que soient les théories auxquelles nous nous sommes référées, nous pouvons constater la médiation corporelle tonique de notre communication avec le monde. Par l'observation croisée nous nous trouvons face à plusieurs interprétations qui peuvent être différentes et cette différence est protectrice.

\section{BIBLIOGRAPHIE}

BARRIER G., « Geste, acte graphique et inférence. La communication au delà du code », thèse, Université Paris 7, 1998.

BERKOWITZ L., A survey of social psychology, Hillsdale III, The Driden Press, 1975.

BOULANGER BALLEYGUIER G., « premières réactions devant le miroir », Enfance 1964, N 1 pp. 51 et 67 et « étapes de la reconnaissance de soi devant le miroir », Enfance 1967, N 1, pp. 91-116.

BIRDWHISTELL R.L. Introduction to kinesics, Université of Louisville Press, 1952.

CASSELL L, SULLIVAN J. \& al., Emboild Conversational Agents Eds, The MIT Press, 2000.

COSNIER J., « Communications et langages gestuels », in J. COSNIER, J. COULON, A. BERENDONNER et C. GREECHIONI Eds, Les voies du langage, Paris, éditions Dunod, 1982.

COSNIER J., A. BROSSARD, La communication non-verbale, Neuchâtel, Delachaux et Niestlé, 1984.

DARWIN C, L'expression des émotions chez l'homme et les animaux, Paris, Reinwald., 1980.

EKMAN P., FRIESEN W.V, Manual for the facial action code, Palo Alto : Consulting Psychologists Press, 1982.

EFRON D., Gesture race and culture, La Haye, Mouton, 1972,

EKMAN P., Hager J-C., Friesen W.V, « Symetry and the narure of facial action », in

Psychophysiology, IS,2, 101-106, 1981. 
FRAISSE, article sur l'émotion Encyclopedia Universalis V6, p. 144, 1990.

GABAY M., Magnétoscope et formation à l'observation des attitudes

corporelles. Contribution à une étude du langage du corps, Thèse, Toulouse Le Mirail, 1978.

GABAY M., Contribution à une étude de la communication interactive et interpersonnelle en situation de face à face, HDR Grenoble 3, 1990. Goffman E., Les rites d'interaction, traduction française A. Kihm, Editions de minuit, 1974.

KENDON A., Studies in the behavior of social interaction, Bloomington, Ind : Indiana University Press, 1977.

LORENZ K., Ausruschsbewegungen Hoerer tiere, Naturwissenschaft, 1951

LE CAMUS J., Contribution ci l'étude de la maladresse chez l'enfant, Paris, Douin, 1975.

LEROY Gouran, Le geste et la parole, technique et langage, col sciences d'aujourd'hui, Albin Michel, pp. 77 et suivantes, 1975 .

LIPPMAN A., «Interactivité », Réseaux. n 33 janv. 1989, CNET.

MAUSS M., 1936, « Communication » (réédité), in sociology and anthropology, Paris, Editions PUF, $3^{\mathrm{e}}$ édition 1966.

MONTAGNER H., L 'enfant et la communication, Paris, éditions Pernoud/Stock, 1978.

ZAJONC R., Psychologie sociale expérimentale, traduction française, Paris éditions Dunod, 1972.

\section{AUTEUR}

MICHÈLE GABAY

CRECI, Université Paris 7 